\title{
Analysis of Offensive Technical Actions on Different Playing Surfaces in 5v5, 7v7 and 9v9 Sided Games in Soccer
}

\author{
Jorge Diaz-Cidoncha Garcia ${ }^{1, *}$, Evelia Franco Álvarez ${ }^{2}$, Alexandre Dellal ${ }^{3}$ \\ ${ }^{1}$ Fédération Internationale de Football Association (FIFA) / Education and Technical Development Department, \\ Niederdorfstrasse 48. 8001 Zurich. Switzerland. \\ ${ }^{2}$ National Institute of Physical Education (INEF), Polytechnic University of Madrid / Sports Department, C/ \\ Martín Fierro, 7. 28040 Madrid, Spain. \\ ${ }^{3}$ FIFA Medical Excellence Centre, Santy. Orthopedicae clinical, sport science and research department, Lyon, \\ France, 24 Avenue Paul Santy. 69008 Lyon, France.
}

*Corresponding Author: Jorge Diaz-Cidoncha Garcia, Fédération Internationale de Football Association (FIFA) / Education and Technical Development Department, Niederdorfstrasse 48. 8001 Zurich. Switzerland.

\begin{abstract}
There are a large number of variables that influence the development of technical actions in soccer. Thus, the objective of this study is to analyse the effects of the surface (grass, turf or dirt) on teams' and players' performances from a technical perspective. Fifty-four games in three different formats $(5 v 5,7 v 7$ and $9 v 9)$ divided into two age groups $(U-9$ and $U-14)$ at three Spanish soccer clubs were filmed and analysed. This study used the observational and descriptive method and was carried out by systematic direct observation, considering different types of behaviour within a set of defined technical offensive variables. Results showed that there were significant differences, and grass pitches generated more attacks (shots per minute: 0.69; touches per game: 258), while turf and dirt pitches generated less offensive play (shots per minute: 0.49 for turf / 0.30 for dirt; touches per game: 224 for turf / 252 for dirt).Conclusion: the data revealed that the characteristics of the playing surface have a strong influence on the development of the players' technical performance.
\end{abstract}

Keywords: Offensive Play, Playing Surfaces, Reduced Spaces, Sided Games, Soccer Technique

\section{INTRODUCTION}

Technical actions in soccer are the movements that are the most suitable for achieving the greatest effect, that is, actions and movements that are most effective and use the least energy [1]. However, a technical skill is not an objective itself but rather a mean to implement the tactical principles of soccer [2]. The most appropriate technical moves will be determined by the situations arising during a match [3]. As each situation is unique, a successful player has the ability to adapt and perform the technical moves that are most appropriate with regard to the playing surface. Previous studies suggest different perceptions between the systems of artificial turf, which generate differences in the physical performance and in the soccer players' performances[4].

Soccer has traditionally been played on grass, which has certain requirements as it is a living plant that changes with the seasons [5]. In some locations, grass grows all year round, while in other locations, the season has an effect on the condition of the grass. Grass requires sunlight and nutrients, it must be looked after and maintained, and it needs regeneration time. Nowadays, it is particularly common to lay instant grass in stadiums rather than sowing grass seed [6]. Nonetheless, grass still requires time and care. Therefore, future studies should look into its influence on the technical variables depending on the quality and the state of the grass.

In recent years, artificial turf has become an accepted playing surface in soccer, as there have been developments in turf specifically designed for the sport. Turf surfaces allow players to move rapidly and safely [7]. However, the player must still adapt to the turf. The uniformity of this type of surface allows a rapid and precise playing style, and offers opportunities to both technically skilled players and those with strong physical attributes. An additional benefit is that artificial pitches can withstand more frequent use than natural pitches, allowing for increased use of the pitch [8]. Additionally, artificial pitches are not greatly affected by the weather conditions (for example rain or snow), while 
grass or dirt pitches can be significantly damaged by the elements. The required regular maintenance (which is essential to keep artificial pitches in optimum condition) is less expensive than maintaining a high-quality grass pitch. For these reasons, turf is now a viable and attractive alternative to grass [9].

Dirt pitches are less common but are still used by amateur and semi-professional clubs. On dirt surfaces, the ball bounces higher than on turf. If the ground is dry and hard, the ball will bounce even higher than usual. While dirt pitches do not require special care or treatment, this type of surface tends to become waterlogged with rain or very hard in cold temperatures. Players are also more likely to become injured playing on dirt pitches [10].

\subsection{Sided Games in Soccer}

Sided games have become increasingly popular in both organized and spontaneous set-ups [11]. This type of format offers a wide variety of possibilities and combinations, and enables a greater amount of interaction between participants. Sided games in soccer are understood to mean $5 \mathrm{v} 5$ (with or without a goalkeeper), $7 \mathrm{v} 7$ and $9 \mathrm{v} 9$ formats. The highest movement regularity found in $4 \mathrm{v} 4$ and $5 \mathrm{v} 5$ identified these formats as more adequate to promote team-related emergent and self-organised behaviours [12].Playing sided soccer has great benefits for players, especially in the learning stages [13]. The size of the field of play is smaller than in 11v11 and the rules are adapted accordingly (using smaller areas and goalposts, for example). Major evidence has shown that players get more time on the ball and make more decisions during the game, increasing the learning process (concentration levels increase because the ball is never far away).

In that sense, the aim of this study is to identify the impact of playing surfaces (turf, grass and dirt) on the technical actions performed in 5v5, 7v7 and 9v9 and its practical implications.

\section{MATERIALS AND METHODS}

\subsection{Participants}

Fifty-four games played by boys in the U-9 and U-14 age groups were used as the sample (378 players for both age categories). The players' physical characteristics were as follows: U-9: height $1.34 \mathrm{~m} \pm 0.12 \mathrm{~m} \mathrm{~cm}$, body mass $29.4 \mathrm{~kg} \pm 11.6 \mathrm{~kg}$; U-14: height $1.63 \mathrm{~m} \pm 0.14 \mathrm{~m}$, body mass $52.9 \mathrm{~kg} \pm$ $13.1 \mathrm{~kg}$. To ensure that the sample was as broad as possible, the same teams were monitored for a specific period of time and all three formats $(5 \mathrm{v} 5,7 \mathrm{v} 7$ and $9 \mathrm{v} 9)$ were taken into account. Games were filmed at three different locations, the academy run by the Spanish Soccer Association (RFEF), and at two different clubs, Adarve-Barrio del Pilar and Villanueva del Pardillo (all teams in the first or second division of the Spanish youth leagues). Of the 54 games in the sample, the following games were filmed (and subsequently analysed): $27 \times 2$ for each age group (U-9 and U-14); $18 \times 3$ on each playing surface (natural grass, artificial turf, dirt); $18 \times 3$ in each game format $(5 \mathrm{v} 5,7 \mathrm{v} 7,9 \mathrm{v} 9)$. The games were 20-minutes long, with no breaks or substitutions. A highly representative sample was selected, considering the main factors that could influence a game's development $\left(24^{\circ}\right.$ external temperature, $60 \%$ humidity) and using the same facility for every match.

\subsection{Procedures}

The observational method was used with the following steps: formulating a problem, collecting and recording the data, analysing and interpreting the observed data and communicating the results [14].All games were watched live on the pitch $(20 \times 30 \mathrm{~m}$. for $5 \mathrm{v} 5,30 \times 45 \mathrm{~m}$. for $7 \mathrm{v} 7,45 \times 60 \mathrm{~m}$ for $9 \mathrm{v} 9$, $24^{\circ}$ external temperature, $60 \%$ humidity) and were subsequently analysed with the aid of the recordings. Measures of reliability required data on categories (surface, game format and age group) rated by three different reviewers. Before playing the match, participants carried out a standard warmup which included exercises such as 5 min of continuous run, 5 min of articulation mobility and two sprints of $20 \mathrm{~m}$. They were not verbally instructed during the games. The technical offensive variables were grouped in touches (any contact with the ball by any legal part of player's body), attacking play (any technical action oriented to overcome the opponent), general play (any action with ball possession) and goalkeepers (any action performed by a goalkeeper) and were measured on each of the surfaces (grass, turf and dirt), through observational and descriptive method carried out by systematic direct observation [15]. This enabled the key technical trends that emerged on each surface type to be analysed, showing any significant effect the surface type had on technical performance in the different combinations of age groups and game formats. The use of human subjects in this research, for the purpose of collecting and statistically analysing the data, was approved by the 
Polytechnic University of Madrid's Institutional Review Board in accordance with the Helsinki declaration of 2013. Assent and parental consent were obtained from all participants.

\subsection{Equipment - Instruments}

The following equipment was used in the sided games: soccer balls (size 4 for U-9 and size 5 for U14), differently-coloured bibs, cones, markers, mini-goals and seven-a-side goals (depending on the game format). A Traceable digital stopwatch was used for timekeeping. For filming purposes, a Sony HDR CX570 camera and a HI-POD tripod were used. A TV monitor was used to watch and analyse the games once all of the planned sessions had been filmed and correctly categorised. Each player had a pitch ratio of $4.8 \mathrm{~m}$ for $5 \mathrm{v} 5,6.4 \mathrm{~m}$ for $7 \mathrm{v} 7$ and $5.5 \mathrm{~m}$ for $9 \mathrm{v} 9$.

\subsection{Statistical Analysis}

All of the games were watched on site and then analysed from the recordings, with the same process being followed at all times. The data was collected as the recordings were watched, with previously defined variables being monitored. The variables were then compared across grass, turf and dirt to establish whether there were any significant variations. Before performing the analyses, inter-rater reliability was confirmed by the calculation of the Cohen Kappa coefficient for each variable under observation. Kappa values were over 0.95 , which are widely accepted as excellent [16].After applying a Saphiro-Wilk test (revealing a normal distribution of the data), the arithmetic mean and standard deviation of each variable observed in the footage of the 54 games was calculated. The distribution of the normality test led to parametric tests. Firstly, a t-test for independent samples was performed in order to find any possible differences in variables scores between U-9 and U-14 groups. After that, two $3 \times 3$ ANOVAs were performed to analyse the effects of surface and format game on technical variables both in U-9 and U-14 groups. Post hoc comparisons were done using the Tukey test ( $<<.05$ ). Partial eta squared ( $\eta \mathrm{p} 2)$ provided an index of effect size. All tests were applied using an SPSS 22.0 pack (Chicago, Illinois, USA).

\subsection{Definition of Variables Considered in the Study}

Following are the definitions of the variables considered in this study [17]:

Total time ball out of play: time whenever the ball is not being played.

Total touches per game: total number of contacts with the ball by any legal part of a player's body, by all players.

Average touches per outfield player: average number of contacts with the ball by any legal part of a player's body, by all outfield players.

Average touches per minute: average number of contacts per minute with the ball by any legal part of a player's body, by all players.

Touches in defensive half: total number of contacts with the ball in their own half by any legal part of a player's body, by all players.

Touches in attacking half: total number of contacts with the ball in the opponent's half by any legal part of a player's body, by all players.

Attempted 1v1 (vs. Goalkeeper): any successful or unsuccessful attempt by a player to take on the opposition goalkeeper directly in attacking play.

Attempts at goal (total): any attempted shot, off and on target.

Attempts at goal (on target): any attempted shot on target, not including hitting the posts or crossbar.

Attempts at goal (off target): any attempted shot that is not on target, including hitting the posts or crossbar.

Average shots per minute: average attempts to score a goal by both teams per minute, made with any (legal) part of the body, either on or off target.

Average goals per minute: average scored goals by both teams per minute.

Total penalty area entries: total number of actions when the ball enters the penalty area under the control of the attacking team.

Number of crosses: total number of balls played from a wide area into the box with the aim of creating a goal scoring opportunity (including corners and free-kicks). A cross must be aimed into the penalty area with the intention of finding a team mate. 
Analysis of Offensive Technical Actions on Different Playing Surfaces in 5v5, 7v7 and 9v9 Sided Games in Soccer

Number of dribbles (total): total attempts by a player to beat an opponent in possession of the ball.

Number of dribbles (unsuccessful): total number of dribbles when the attacker is tackled by the defender.

Number of dribbles (successful): total number of dribbles when the attacking player beats the defender while retaining possession.

Attempted passes (total): total number of intentional played balls from one player to another.

Goalkeeper (touches): all of the below goalkeeper events are also coded as a goalkeeper touches.

Goalkeeper (saves): any type of goal attempt caught/stopped/blocked by the goalkeeper.

Goalkeeper (kicks): a ball kicked by the goalkeeper in open play.

Goalkeeper (throws): a throw by the goalkeeper intending to reach a specific target.

Goal kick: ball kicked back into play by the goalkeeper (following the ball going out of play by the by-line).

\section{RESUlts}

\subsection{Descriptive Statistics and Comparison according to Category}

Descriptive statistics and t-test for independent samples are showed in Table 1. Scores were significantly higher among U-9 group in total time ball out of play $(t=2.27 ; p=<05)$, total penalty area entries $(\mathrm{t}=2.55 ; \mathrm{p}=\langle 05)$, number of dribbles (total)* $(\mathrm{t}=2.16 ; \mathrm{p}=\langle 05)$, while they were significantly higher among $\mathrm{U}-14$ group in touches in defensive half $(\mathrm{t}=-2.22 ; \mathrm{p}=\langle 05)$ and attempted passes $(\mathrm{t}=-2.86 ; \mathrm{p}=<05)$.

Table 1. Means and standard deviations in study variables for $U-9$ and U-14 groups

\begin{tabular}{|c|c|c|}
\hline \multirow{2}{*}{ Variables } & \multicolumn{2}{|c|}{ M (SD) } \\
\hline & U-9 & U-14 \\
\hline \multicolumn{3}{|c|}{ Ball out of play } \\
\hline Total time ball out of play* & $430.93 "\left(85.79^{\prime \prime}\right)$ & $387.59^{\prime \prime}\left(49.83^{\prime \prime}\right)$ \\
\hline \multicolumn{3}{|c|}{ Touches } \\
\hline Total touches per game & $233.56(51.24)$ & $256.30(57.76)$ \\
\hline Average touches per outfield player & $40.78(17.99)$ & $42.07(16.16)$ \\
\hline Average touches per minute & $11.22(3.13)$ & $12.41(3.86)$ \\
\hline Touches in defensive half* & $121.67(26.15)$ & $140.85(36.44)$ \\
\hline Touches in attacking half & $111.52(33.68)$ & $118.04(40.56)$ \\
\hline \multicolumn{3}{|c|}{ Attacking Play } \\
\hline Attempted 1v1 (vs. Goalkeeper) & $0.56(0.97)$ & $0.26(0.53)$ \\
\hline Attempts at goal (total) & $8.85(4.77)$ & $7.30(3.41)$ \\
\hline Attempts at goal (on target) & $5.78(3.67)$ & $4.74(2.41)$ \\
\hline Attempts at goal (off target) & $3.07(2.00)$ & $2.56(1.60)$ \\
\hline Average shots per minute & $0.63(.96)$ & $0.39(.26)$ \\
\hline Average goals per minute & $0.12(0.10)$ & $0.11(0.06)$ \\
\hline Total penalty area entries* & $13.22(4.55)$ & $10.44(3.37)$ \\
\hline \multicolumn{3}{|c|}{ General Play } \\
\hline Number of crosses & $4.22(2.03)$ & $3.44(2.12)$ \\
\hline Number of dribbles (total)* & $11.74(3.81)$ & $9.78(2.78)$ \\
\hline Number of dribbles (unsuccessful) & $3.22(1.85)$ & $2.41(1.31)$ \\
\hline Number of dribbles (successful) & $8.52(2.99)$ & $7.37(2.13)$ \\
\hline Attempted passes (Total) & $66.22(16.15)$ & $79.33(17.50)$ \\
\hline \multicolumn{3}{|c|}{ Goal Keeper } \\
\hline Goalkeepers (touches) & $11.37(5.71)$ & $12.70(5.61)$ \\
\hline \begin{tabular}{|l} 
Goalkeepers (saves) \\
\end{tabular} & $3.22(2.68)$ & $3.37(2.53)$ \\
\hline Goalkeepers (kicks) & $3.96(2.78)$ & $4.70(3.35)$ \\
\hline Goalkeepers (throws) & $1.78(2.38)$ & $2.04(1.81)$ \\
\hline Goal kicks & $4.22(2.53)$ & $\begin{array}{ll}3.85 & 1.68) \\
\end{array}$ \\
\hline
\end{tabular}

\subsection{Effects of Surface and Game Format on Study Variables for the U-9 Group}

The descriptive statistics for study variables in U-9 group in each of the surfaces are shown in Table 2. The two-way ANOVA showed a main effect for surface in total time ball out of play $(\mathrm{F}=10.30 ; \mathrm{p}$ $<.01 ; \eta 2=0.53)$, total touches per game $(\mathrm{F}=14.46 ; \mathrm{p}<.01 ; \eta 2=0.62)$, average touches per outfield player $(F=11.73 ; p<.01 ; \eta 2=0.57)$, average touches per minute $(F=6.77 ; p<.01 ; \eta 2=0.43)$, touches 
Analysis of Offensive Technical Actions on Different Playing Surfaces in 5v5, 7v7 and 9v9 Sided Games in Soccer

in defensive half $(F=26.44 ; p<.01 ; \eta 2=0.75)$, average shots per minute $(F=4.16 ; p<.05 ; \eta 2=0.32)$, total number of dribbles $(F=7.92 ; p<.01 ; \eta 2=0.47)$, number of unsuccessful dribbles $(F=3.60 ; p$ $<.05 ; \eta 2=0.29)$, number of successful dribbles $(\mathrm{F}=5.38 ; \mathrm{p}<.05 ; \eta 2=0.37)$ and goal kicks $(\mathrm{F}=4.62$; $\mathrm{p}<.05 ; \eta 2=0.34)$.

Table2. Means and standard deviations in study variables according to surface

\begin{tabular}{|c|c|c|c|c|c|c|}
\hline & \multicolumn{3}{|c|}{ U-9 } & \multicolumn{3}{|c|}{ U-14 } \\
\hline & Dirt & Grass & Turf & Dirt & Grass & Turf \\
\hline & M (SD) & M (SD) & M (SD) & M (SD) & M (SD) & M (SD) \\
\hline \multicolumn{7}{|c|}{ Ball out of play } \\
\hline \multirow{2}{*}{ Total time ball out of play ${ }^{1}$} & 371.11" & $422.44 "$ & 499.22 & $410.00 "$ & $377.78^{\prime \prime}$ & $375.00 "$ \\
\hline & $(65.52 ")$ & $(69.88 ")$ & $\left(73.40^{\prime \prime}\right)$ & $(58.79 ")$ & $(45.56 ")$ & $(41.08 ")$ \\
\hline \multicolumn{7}{|c|}{ Toques } \\
\hline \multirow{2}{*}{ Total touches per game ${ }^{1}$} & 271.44 & 241.22 & 188.00 & 233.44 & 275.22 & 260.22 \\
\hline & $(41.00)$ & $(45.46)$ & $(27.76)$ & $(28.49)$ & $(86.09)$ & $(40.13)$ \\
\hline \multirow{2}{*}{ Average touches per outfield player ${ }^{1}$} & 47.56 & 42.33 & 32.44 & 39.78 & 45.44 & 41.00 \\
\hline & $(18.78)$ & $(20.63)$ & $(11.89)$ & $(15.22)$ & $(13.99)$ & $(20.02)$ \\
\hline \multirow{2}{*}{ Average touches per minute ${ }^{1}$} & 13.44 & 10.78 & 9.44 & 11.22 & 14.22 & 11.78 \\
\hline & $(1.88)$ & $(4.12)$ & $(1.42)$ & $(1.30)$ & $(4.15)$ & $(4.87)$ \\
\hline \multirow{2}{*}{ Touches in defensive half } & 142.22 & 123.89 & 98.89 & 127.56 & 153.78 & 141.22 \\
\hline & $(13.72)$ & $(21.32)$ & $(22.70)$ & $(18.17)$ & $(41.72)$ & $(43.11)$ \\
\hline \multirow{2}{*}{ Touches in attacking half } & 118.11 & 117.33 & 99.11 & 102.56 & 132.56 & 119.00 \\
\hline & $(38.17)$ & $(38.49)$ & (22.08) & $(27.08)$ & $(56.28)$ & $(30.64)$ \\
\hline \multicolumn{7}{|c|}{ Attacking Play } \\
\hline \multirow{2}{*}{ Attempted 1 vs 1 (vs. Goalkeeper) } & 0.11 & 0.44 & 1.11 & 0.33 & 0.22 & 0.22 \\
\hline & $(0.33)$ & $(1.01)$ & $(1.17)$ & $(0.71)$ & $(0.44)$ & $(0.44)$ \\
\hline \multirow{2}{*}{ Attempts at goal (total) } & 8.44 & 8.56 & 9.56 & 5.78 & 8.33 & 7.78 \\
\hline & $(6.80)$ & $(4.67)$ & $(2.24)$ & $(2.82)$ & $(3.74)$ & $(3.42)$ \\
\hline \multirow{2}{*}{ Attempts at goal (on target) } & 5.00 & 5.67 & 6.67 & 4.33 & 5.00 & 4.89 \\
\hline & $(4.36)$ & $(4.12)$ & $(2.50)$ & $(2.40)$ & $(2.87)$ & $(2.15)$ \\
\hline \multirow{2}{*}{ Attempts at goal (off target) ${ }^{2}$} & 3.44 & 2.89 & 2.89 & 1.44 & 3.33 & 2.89 \\
\hline & $(2.60)$ & $(1.62)$ & $(1.83)$ & $(1.51)$ & $(1.12)$ & $(1.62)$ \\
\hline \multirow{2}{*}{ Average shots per minute ${ }^{1}$} & 0.41 & 0.95 & 0.53 & 0.28 & 0.44 & 0.45 \\
\hline & $(0.32)$ & $(1.63)$ & $(0.22)$ & $(0.14)$ & $(0.19)$ & $(0.39)$ \\
\hline \multirow{2}{*}{ Total penalty area entries } & 11.56 & 14.11 & 14.00 & 9.56 & 9.56 & 12.22 \\
\hline & $(5.98)$ & $(4.57)$ & $(2.45)$ & $(1.74)$ & $(3.36)$ & $(4.15)$ \\
\hline \multicolumn{7}{|c|}{ General play } \\
\hline \multirow{2}{*}{ Number of crosses } & 4.44 & 4.11 & 4.11 & 2.67 & 4.78 & 2.89 \\
\hline & $(2.30)$ & $(2.26)$ & $(1.69)$ & $(1.73)$ & $(2.33)$ & $(1.76)$ \\
\hline \multirow{2}{*}{ Number of dribbles (total) $)^{1,2}$} & 10.89 & 15.00 & 9.33 & 8.89 & 10.56 & 9.89 \\
\hline & $(2.93)$ & $(2.60)$ & $(3.54)$ & $(3.14)$ & $(1.67)$ & $(3.30)$ \\
\hline Number of dribbles (uncuccessful) 1 & 3.44 & 4.11 & 2.11 & 2.11 & 3.11 & 2.00 \\
\hline & $(1.33)$ & $(2.26)$ & $(1.36)$ & $(1.05)$ & $(0.78)$ & $(1.73)$ \\
\hline Number of dribbles (successful) 1 & 7.44 & 10.89 & 7.22 & 6.78 & 7.44 & 7.89 \\
\hline & $(2.88)$ & $(1.83)$ & $(2.82)$ & $(2.39)$ & $(1.59)$ & $(2.42)$ \\
\hline Attemnted noces (total) & 74.67 & 65.11 & 58.89 & 67.67 & 82.78 & 87.56 \\
\hline Attempted passes (total) & $(19.07)$ & $(11.10)$ & (14.80) & $(9.30)$ & $(17.75)$ & $(18.75)$ \\
\hline & & Goal K & & & & \\
\hline Tanol 1,2 & 12.67 & 12.44 & 9.00 & 14.44 & 11.00 & 12.67 \\
\hline Touches & $(5.74)$ & $(6.06)$ & $(5.15)$ & $(5.59)$ & $(5.61)$ & $(5.72)$ \\
\hline Saves 1,2 & 3.00 & 4.67 & 2.00 & 5.22 & 2.44 & 2.44 \\
\hline Saves & $(1.66)$ & (3.12) & $(2.60)$ & $(3.19)$ & $(1.67)$ & $(1.42)$ \\
\hline & 5.78 & 2.78 & 3.33 & 4.33 & 4.00 & 5.78 \\
\hline Kicks & $(3.07)$ & (1.64) & (2.69) & $(3.12)$ & $(2.60)$ & $(4.24)$ \\
\hline & 2.22 & 2.78 & 0.33 & 2.67 & 1.89 & 1.56 \\
\hline Throws & $(2.59)$ & (2.77) & $(0.50)$ & $(2.06)$ & $(1.54)$ & (1.81) \\
\hline Gool kicko & 4.67 & 4.33 & 3.67 & 4.33 & 3.56 & 3.67 \\
\hline Goal kicks ${ }^{1}$ & $(1.58)$ & (2.74) & $(3.20)$ & $(2.00)$ & $(1.67)$ & $(1.41)$ \\
\hline
\end{tabular}

Note: ${ }^{l}=$ Significant differences were found in $U-9$ group ${ }^{2}=$ significant differences were found in $U-14$ group. Post-hoc test results are summarized in Table 3. 
Analysis of Offensive Technical Actions on Different Playing Surfaces in 5v5, 7v7 and 9v9 Sided Games in Soccer

Table3. Summary of differences found in study variables according to surface and game format

\begin{tabular}{|c|c|c|c|c|c|c|}
\hline \multirow[b]{2}{*}{ Variables } & \multicolumn{3}{|c|}{ U-9 } & \multicolumn{3}{|c|}{ U-14 } \\
\hline & Surface & Format & Int. & Surface & Format & Int. \\
\hline Ball out of play & Turf $>$ Dirt, Grass & & & & & \\
\hline Total touches per game & Turf $>$ Dirt, Grass & $5 v 5>7 v 7,9 v 9$ & & & & \\
\hline $\begin{array}{l}\text { Average touches per } \\
\text { outfield player }\end{array}$ & Turf $>$ Dirt,Grass & $5 \mathrm{v} 5>7 \mathrm{v} 7,9 \mathrm{v} 9$ & & & $5 v 5>7 v 7,9 v 9$ & \\
\hline $\begin{array}{lll}\begin{array}{l}\text { Average touches } \\
\text { minute }\end{array} & \\
\end{array}$ & Turf > Dirt & $5 v 5>7 v 7$ & & & & \\
\hline Touches in defensive half & & & $* 1$ & & & \\
\hline Touches in attacking half & & $5 v 5>7 v 7$ & & & & \\
\hline Attempted 1 vs. 1 & & & & & & \\
\hline Attempts at goal & & $5 \mathrm{v} 5>7 \mathrm{v} 7,9 \mathrm{v} 9$ & & & & \\
\hline $\begin{array}{l}\text { Attempts at goal (on } \\
\text { target) }\end{array}$ & & $5 \mathrm{v} 5>7 \mathrm{v} 7,9 \mathrm{v} 9$ & & & & \\
\hline $\begin{array}{l}\text { Attempts at goal (off } \\
\text { target) }\end{array}$ & & & & Dirt $>$ Grass & & \\
\hline Total goals & Grass $>$ Turf & $5 \mathrm{v} 5>7 \mathrm{v} 7,9 \mathrm{v} 9$ & & & $5 v 5>9 v 9$ & \\
\hline Average goals per minute & Grass $>$ Turf & $5 v 5>7 v 7,9 v 9$ & & & $5 v 5>9 v 9$ & \\
\hline Total penalty areas entries & & $5 v 5>7 v 7,9 v 9$ & & & & \\
\hline Number of dribbles (total) & $\begin{array}{c}\text { Grass }>\text { Dirt, } \\
\text { Turf }\end{array}$ & & & & $7 \mathrm{v} 7>5 \mathrm{v} 5,9 \mathrm{v} 9$ & \\
\hline Unsuccessful dribbles & Grass > Turf & & & & & \\
\hline Successful dribles & $\begin{array}{c}\text { Grass }>\text { Dirt, } \\
\text { Turf } \\
\end{array}$ & & & & $7 \mathrm{v} 7>5 \mathrm{v} 5,9 \mathrm{v} 9$ & \\
\hline Attempted passes & & & & Turf $>$ Dirt & & \\
\hline Goalkeeper touches & & $5 v 5>7 v 7>9 v 9$ & & & $5 v 5>9 v 9$ & \\
\hline Goalkeeper saves & & $5 v 5>9 v 9$ & & $\begin{array}{c}\text { Dirt }>\text { Grass, } \\
\text { Turf }\end{array}$ & $5 v 5,7 v 7>9 v 9$ & \\
\hline Goalkeeper kicks & Dirt $>$ Grass & $5 v 5>9 v 9$ & & & & \\
\hline Goalkeepers throws & Grass $>$ Turf & $5 v 5>9 v 9$ & & & & \\
\hline Goal kicks & & & & & $5 v 5>9 v 9$ & \\
\hline
\end{tabular}

The descriptive statistics for study variables in the U-9 group in each of the game format are shown in Table 4. The two-way ANOVA showed a main effect for game format in total touches per game $(\mathrm{F}=$ $5.39 ; \mathrm{p}<.05 ; \eta 2=0.37)$, average touches per outfield player $(\mathrm{F}=67.88 ; \mathrm{p}<.01 ; \eta 2=0.88)$, average touches per minute $(F=4.97 ; p<.05 ; \eta 2=0.36)$, touches in attacking half $(F=4.48 ; p<.05 ; \eta 2=$ $0.33)$, attempts at goal $(F=9.62 ; p<.01 ; \eta 2=0.52)$, attempts at goal on target $(F=12.50 ; p<.01 ; \eta 2=$ $0.58)$, average shots per minute $(F=11.20 ; p<.01 ; \eta 2=0.55)$, total penalty area entries $(F=11.22 ; p$ $<.01 ; \eta 2=0.55)$, goalkeeper touches $(F=21.18 ; \mathrm{p}<.01 ; \eta 2=0.70)$, saves $(F=4.31 ; p<.05 ; \eta 2=0.32)$; $\operatorname{kicks}(\mathrm{F}=3.81 ; \mathrm{p}<.05 ; \eta 2=0.30)$ and throws $(\mathrm{F}=5.75 ; \mathrm{p}<.05 ; \eta 2=0.39)$.

There was a significant interaction effect between surface and game format on touches in defensive half as it is shown in Table 3.

\subsection{Effects of Surface and Game Format on Study Variables for U-14 Group}

The descriptive statistics for study variables in the U-14 group in each of the surfaces are shown in Table 2. The two-way ANOVA showed a main effect for attempts at goal (off target) $(\mathrm{F}=5.39 ; \mathrm{p}$ $<.05 ; \eta 2=0.37)$, total attempted passes $(F=3.71 ; p<.05 ; \eta 2=0.29)$, goalkeeper touches $(F=7.44 ; p$ $<.01 ; \eta 2=0.45)$ and saves $(\mathrm{F}=7.10 ; \mathrm{p}<.01 ; \eta 2=0.44)$.

Post-hoc test results are summarized in Table 3.The descriptive statistics for study variables in the U14 group in each of the game format are shown in Table 4. The two-way ANOVA showed a main effect for game format in average touches per outfield player $(F=10.35 ; p<.01 ; \eta 2=0.53)$, average touches per minute $(F=4.40 ; p<.05 ; \eta 2=0.33)$, total number of dribbles $(F=7.60 ; p<.01 ; \eta 2=0.46)$, 
Analysis of Offensive Technical Actions on Different Playing Surfaces in 5v5, 7v7 and 9v9 Sided Games in Soccer

number of successful dribbles $(F=7.96 ; p<.01 ; \eta 2=0.47)$ goalkeeper touches $(F=7.44 ; p<.01 ; \eta 2=$ $0.45)$, saves $(\mathrm{F}=6.32 ; \mathrm{p}<.01 ; \eta 2=0.41)$ and goal kicks $(\mathrm{F}=3.92 ; \mathrm{p}<.05 ; \eta 2=0.30)$.

No interaction effects were found in this category. Post-hoc test results are summarized in Table 3.

Table4. Means and standard deviations in study variables according to game format

\begin{tabular}{|c|c|c|c|c|c|c|}
\hline \multirow{3}{*}{ Variables } & \multicolumn{3}{|c|}{ U-9 } & & U-14 & \multirow[b]{2}{*}{ 9v9 } \\
\hline & $5 v 5$ & $7 \mathrm{v} 7$ & 9v9 & $5 \mathrm{v} 5$ & $7 \mathrm{v} 7$ & \\
\hline & M (SD) & M (SD) & M (SD) & M (SD) & M (SD) & M (SD) \\
\hline \multicolumn{7}{|c|}{ Ball out of play } \\
\hline \multirow{2}{*}{ Total time ball out of play } & 403.33" & $472.78^{\prime \prime}$ & 416.67" & $400.56 "$ & $380.56 "$ & $381.67 "$ \\
\hline & $\left(88.88^{\prime \prime}\right)$ & $(84.52 ")$ & (76.27") & $(52.76 ")$ & $(60.90 ")$ & (35.97") \\
\hline \multicolumn{7}{|c|}{ Toques } \\
\hline \multirow{2}{*}{ Total touches per game ${ }^{1}$} & 263.00 & 214.89 & 222.78 & 242.67 & 246.67 & 279.56 \\
\hline & $(57.76)$ & $(49.03)$ & $(36.01)$ & $(60.92)$ & $(27.95)$ & $(73.68)$ \\
\hline \multirow{2}{*}{ Average touches per outfield player ${ }^{1,2}$} & 61.78 & 33.44 & 27.11 & 56.56 & 39.22 & 30.44 \\
\hline & $(14.12)$ & $(8.22)$ & $(4.26)$ & $(14.34)$ & $(5.14)$ & $(14.81)$ \\
\hline \multirow{2}{*}{ Average touches per minute ${ }^{1}$} & 13.22 & 10.00 & 10.44 & 12.22 & 12.33 & 12.67 \\
\hline & $(2.64)$ & $(3.32)$ & $(2.65)$ & $(3.23)$ & $(1.80)$ & $(5.87)$ \\
\hline \multirow{2}{*}{ Touches in defensive half ${ }^{1}$} & 131.78 & 120.33 & 112.89 & 152.78 & 124.78 & 145.00 \\
\hline & $(21.87)$ & $(25.01)$ & $(30.24)$ & $(35.77)$ & $(18.54)$ & $(47.15)$ \\
\hline \multirow{2}{*}{ Touches in attacking half ${ }^{1}$} & 131.22 & 93.44 & 109.89 & 99.89 & 120.78 & 133.44 \\
\hline & $(38.30)$ & $(23.61)$ & $(29.24)$ & $(24.22)$ & $(24.00)$ & $(59.49)$ \\
\hline \multicolumn{7}{|c|}{ Attacking Play } \\
\hline \multirow{2}{*}{ Attempted 1 vs 1 (vs. Goalkeeper) } & 0.89 & 0.33 & 0.44 & 0.22 & 0.33 & 0.22 \\
\hline & $(1.17)$ & $(0.71)$ & $(1.01)$ & $(0.44)$ & $(0.50)$ & $(0.67)$ \\
\hline \multirow{2}{*}{ Attempts at goal (total) ${ }^{1}$} & 13.22 & 6.56 & 6.78 & 8.44 & 7.78 & 5.67 \\
\hline & $(4.82)$ & $(3.50)$ & $(2.49)$ & $(2.01)$ & $(3.42)$ & $(4.15)$ \\
\hline \multirow{2}{*}{ Attempts at goal (on target) ${ }^{1}$} & 9.22 & 4.00 & 4.11 & 5.44 & 5.11 & 3.67 \\
\hline & $(3.35)$ & $(3.28)$ & $(1.36)$ & $(2.30)$ & $(1.83)$ & $(2.87)$ \\
\hline \multirow{2}{*}{ Attempts at goal (off target) } & 4.00 & 2.56 & 2.67 & 3.00 & 2.67 & 2.00 \\
\hline & $(2.60)$ & $(1.59)$ & $(1.50)$ & $(1.12)$ & $(1.94)$ & $(1.66)$ \\
\hline \multirow{2}{*}{ Average shots per minute ${ }^{1,2}$} & 0.66 & 0.37 & 0.87 & 0.43 & 0.45 & 0.29 \\
\hline & $(0.22)$ & $(0.29)$ & $(1.65)$ & $(0.10)$ & $(0.39)$ & $(0.21)$ \\
\hline \multirow{2}{*}{ Total penalty area entries ${ }^{1}$} & 17.33 & 11.67 & 10.67 & 10.33 & 11.44 & 9.56 \\
\hline & $(3.71)$ & $(3.81)$ & $(3.16)$ & $(3.04)$ & $(4.10)$ & $(2.96)$ \\
\hline \multicolumn{7}{|c|}{ General play } \\
\hline Number of $\mathrm{c}$ & 5.00 & 3.56 & 4.11 & 3.22 & 3.44 & 3.67 \\
\hline Number of crosses & $(2.45)$ & $(1.59)$ & $(1.90)$ & $(2.77)$ & $(1.51)$ & $(2.12)$ \\
\hline Number of dribbles (total) $)^{2}$ & 12.67 & 10.56 & 12.00 & 8.89 & 12.00 & 8.44 \\
\hline Number or aribbies (total) & $(4.69)$ & $(3.40)$ & $(3.32)$ & $(1.83)$ & $(2.55)$ & $(2.60)$ \\
\hline Number of dribblec (uncuccecsful) & 3.67 & 3.00 & 3.00 & 2.00 & 2.89 & 2.33 \\
\hline Number of dribbles (unsuccessful) & $(2.12)$ & $(1.58)$ & $(1.94)$ & $(1.12)$ & $(1.54)$ & $(1.22)$ \\
\hline Number of dribble (curcesful ${ }^{2}$ & 9.00 & 7.56 & 9.00 & 6.89 & 9.11 & 6.11 \\
\hline Number of dribbles (successful) & $(3.50)$ & $(2.79)$ & $(2.74)$ & $(1.62)$ & $(1.90)$ & $(1.76)$ \\
\hline Attemnted nocece (totol) & 71.11 & 64.44 & 63.11 & 79.78 & 77.33 & 80.89 \\
\hline Attempted passes (total) & $(22.08)$ & $(11.08)$ & $\begin{array}{l}(14.01) \\
\end{array}$ & $(20.25)$ & (15.54) & $\begin{array}{l}(18.35) \\
\end{array}$ \\
\hline & Goal & Keeper & & & & \\
\hline Touchec1,2 & 16.78 & 11.00 & 6.33 & 17.11 & 12.44 & 8.56 \\
\hline louches & $\begin{array}{l}(4.79) \\
\end{array}$ & $(3.43)$ & $(3.08)$ & $(4.43)$ & $(5.88)$ & $(2.60)$ \\
\hline Saves 1,2 & 4.89 & 3.11 & 1.67 & 4.56 & 3.89 & 1.67 \\
\hline & $\begin{array}{l}(3.48) \\
\end{array}$ & $\begin{array}{l}(2.03) \\
\end{array}$ & $(1.12)$ & $\begin{array}{l}(2.07) \\
\end{array}$ & $\begin{array}{l}(3.06) \\
\end{array}$ & $(1.41)$ \\
\hline Kicks 1 & 5.33 & 4.11 & 2.44 & 5.67 & 4.56 & 3.89 \\
\hline & $(3.94)$ & $(1.69)$ & $(1.42)$ & $(3.32)$ & $(4.25)$ & $(2.37)$ \\
\hline Throws ${ }^{1}$ & 3.00 & 2.11 & 0.22 & 3.00 & 2.11 & 1.00 \\
\hline Throws & $(2.50)$ & $(2.71)$ & $(0.44)$ & $(2.18)$ & $(1.69)$ & $(0.87)$ \\
\hline Goal kicks 1,2 & 5.56 & 4.00 & 3.11 & 5.00 & 3.44 & 3.11 \\
\hline & $\begin{array}{l}(2.13) \\
\end{array}$ & $(2.40)$ & $(2.67)$ & $(1.12)$ & $\begin{array}{l}(2.07) \\
\end{array}$ & $(1.17)$ \\
\hline
\end{tabular}

Note: ${ }^{1}=$ Significant differences were found in U-9 group; ${ }^{2}=$ significant differences were found in U-14 group 


\section{DISCUSSION}

The games were analysed according to a predefined set of variables that represented a detailed list of the different technical elements that could occur in each game. The differences for each age group according to game format and different surface types, were observed. In general terms (see Table 3), a significant interaction effect between game format and surface type was not found. However, format game resulted to have an important effect on technical variables. This influence was especially important in the number of touches and attempts of goal among the U-9 group. The effect of surface type on technical variables was also more relevant in U-9 than in the U-14 group.

Grass pitches generated a greater level of attacking play, with higher levels of positive data in almost all the variables. On all surfaces there were more attacks (goals, shots on goal, one-on-ones and entries into the penalty area) in the smaller-sided game formats (5v5 and 7v7) than in 9v9 games, which showed a tendency to offensive play in smaller formats. Despite the average number of touches per game by each player was higher on dirt, a greater average number of attempted attacks were seen on turf and grass. Moreover, the number of touches per outfield player was considerably higher in the $5 \mathrm{v} 5$ game format than in the 9v9 format across all surfaces and both age groups. This trend was also observed in the case of goalkeepers, which showed the positive impact of grass surfaces on offensive play.

Matches played on turf resulted in the outfield players having fewer overall touches, which resulted on a more direct attacking play. This data related directly to attempts on goal, one-on-ones, goals scored and entries into the penalty area [18]. It was also observed that, of the attempts at goal across all age groups and formats, $67 \%$ were successful. As was the case for both dirt and grass pitches, there were generally more touches in the defensive half of the pitch for each game format and age group, although the U-9 9v9 and U-14 7v7 games reversed this trend [19]. This data revealed a tendency towards combinative play for both dirt and grass, while turf had fewer touches in the own half (leading to more direct attacking play). There was a higher number of dribbles than crosses across all formats and age groups, revealing a preference of individual actions over collective play. However, the largest variation was in relation to the number of passes. The U-14 group made more passes (an average of 88) than the U-9s (an average of 59). Furthermore, of these attempted passes, a significantly higher percentage was successful in the U-14 age group (83\%) than in the U-9 age group (73\%). Such trends revealed a better understanding of the collective play in the U-14 group, as well as higher passing lines and support from the team mates too.

In line with the trend for both the dirt and grass surface types, the average number of goalkeeper touches decreased as the number of players per team increased [20]. This showed how the relevance of goalkeeper decreased with bigger formats, while it increased in smaller game formats for grass and turf. As with the data for dirt pitches, the notable trend was that the average number of touches per outfield player was generally higher for the smaller the game formats [21, 22]. This showed that the relevance of the goalkeepers in attacking play was higher when the space was reduced (mainly when building the attack, in the initial phase of play).

With respect to the general data (crosses, dribbles, passes), the overall results were similar across all surface types, age groups and formats compared with previous studies [23]. Specifically, the average numbers of crosses and dribbles did not vary significantly on the different surface types [24]. Generally, the number of passes was higher in the U-14 group than in the U-9 group. This showed a more elaborated build-up of the attacks by the U-14 group, by using collective play to overcome the defensive lines of the opponent. However, significant differences were observed for each of the surfaces. On dirt, there were significantly more passes in the U-9 5v5 games than for any other game format or age group (91 for U-9 5v5 compared with an average of 67 for the other age groups and game formats). On grass, there were more passes across both age groups in the $9 \mathrm{v} 9$ game format than for the other formats (average of 85 in $9 \mathrm{v} 9$ compared with 69 in the other formats). On turf, there were a far higher number of passes in the U-14 age group (average 88) than in the U-9 age group (average 59). The highest number of passes in the U-9 age group was on dirt pitches, whereas the highest number for the U-14 age group was on artificial pitches. This trend showed a different behaviour from both age groups in collective play, being turf the most favourable one for U-14 while dirt was for U-9. This tendency showed that different surfaces can build up confidence on the players, when working on collective play. 
The number of touches per game was fairly consistent across all age groups and surfaces [25]. When split by age group, a general trend towards fewer touches per outfield player on turf was evident [26], which revealed the relevance of age groups when analysing ball possession and control. This trend was most noticeable for the U-9 age group, as it focuses mainly on individual play. Although the variables analysed showed that the ball was out of play for more time and that players had fewer touches on turf, it was also observed that there were more attempts at goal on this type of surface than on grass or dirt [27], revealing again the nature of turf when building direct attacks.

Differences were observed in the general data from the two different age groups: in the U-9 age group, the highest number of passes was on dirt and the lowest on turf, while in the U-14 the mentioned variables had opposite results. In both age groups, goalkeepers had more touches on dirt and fewer on turf. This trend was consistent in the U-9 age group, while in the U-14 age group, the lowest number of touches was on grass. On dirt pitches, it is more difficult to control the ball and adapting takes longer, so it is better to pass the ball directly to a teammate's foot. If the ground is irregular, ball control is difficult and takes time, and the bounce of the ball may give rise to unpredictable situations in which it is important to pay close attention [28].

It was observed that the time that the ball spent out of play was broadly consistent, with relatively little variation across each of the game formats and age groups [29]. Such tendency reveals that, besides the actual data presented in this study, any player would need to adapt and make its own decisions in each specific game situation. The total number of touches was also consistent across each game format and age group. However, in line with the general trend on each of the individual surface types, the average number of touches per outfield player across both age groups was highest in the $5 \mathrm{v} 5$ game format and lowest in the $9 \mathrm{v} 9$ format [30]. Once again, this trend showed the positive impact of smaller formats in the learning stages of footballas there are more touches of the ball and more decision-making situations. The number of goalkeeper touches was highest in the $5 \mathrm{v} 5$ game format and lowest in the $9 \mathrm{v} 9$ game format across both age groups and on all surface types. Generally speaking, there was little variation in the general data between the different formats and age groups, confirming that the type of playing surface affected the technical moves performed.

\section{CONCLUSIONS AND PRACTICAL IMPLICATIONS}

This study provides an insight into relationships between game format and surface type with offensive technical outcomes in soccer. However, a potential limitation of this work was that influence of other factors which could have an impact on technical variables were not considered. In addition, the sample size of the observed small sided games was limited and the results could be due to the possible variations in the observations across games rather than the considered playing surfaces. Future studies should address the influence of other factors such as training characteristics, weather, competition, coach behavioural patterns on both technical and tactical performances.

The characteristics of the playing surface have a strong influence on the development of the players' technical performance, as well as on the general development of the game [31]. At the youth level, physical preparation should also be taken into account, as wet or soft ground (as could be the case with grass or dirt pitches) requires greater demand on specific muscles than a pitch with normal conditions [32].

Regarding technical moves, on more slippery ground (grass, turf) the ball picks up speed as soon as it touches the ground [33], making it harder to control. Players who are technically skilled and agile will have an advantage over slower players, as will attack players who take the initiative [34]. Ground level shots on goal will also be difficult for the goalkeeper to control. On grass and turf, technically skilled teams will be favoured. Transitions will be quicker and the game will sometimes seem very fast-paced [35].

On slow and rough ground (grass, dirt), the ball can occasionally stop unexpectedly, making it difficult to build up to and execute technical moves with precision [36]. Long aerial passes are preferred in such cases in order to avoid risky duels and difficulties executing technical moves. A pitch in this condition gives an advantage to athletic players who have to draw on their physical strengths [37]. 
When preparing a session or a game, soccer coaches must consider not only the characteristics and level of their players but also the type of playing surface and its influence on the performance of the technical variables. It is hoped that the objective analysis provided by this study will provide relevant information for interested parties working in soccer development.

\section{REFERENCES}

[1] Fédération Internationale de Football Association. Coaching manual. Zurich: FIFA Education and Technical Development, 2013.

[2] Lago-Peñas, C., Casais, L., Dellal, A., Rey, E., \& Domínguez, E. Anthropometric and physiological characteristics of young soccer players according to their playing positions: relevance for competition success. Journal of Strength and Conditioning Research, 2011, 25, 3358-3367.

[3] Castagna, C., Belardinelli, R., Impellizzeri, F.M., Abt, G. A., Coutts, A. J., \& D'ottavio, S. Cardiovascular responses during recreational 5-a-side indoor-soccer. Journal of Science and Medicine in Sport, 2007, 10, 89-95.

[4] Sánchez-Sánchez, J., García-Unanue, J., Jiménez-Reyes, P., Gallardo, A., Burillo, P., Felipe, J.L., \& Gallardo, L. Influence of the Mechanical Properties of Third-Generation Artificial Turf Systems on Soccer Players' Physiological and Physical Performance and Their Perceptions.PLoS ONE, 2014, 9, 1-11.

[5] Sassi, A., Stefanescu, A., Menaspa, P., Bosio, A., Riggio, M., \& Rampinini, E. The cost of running on natural grass and artificial turf surfaces. Journal of Strength and Conditioning Research, 2011, 25, 606611.

[6] Fédération Internationale de Football Association. Manager's guide to natural grass football pitches. Zurich: FIFA Member Associations and Development, 2010.

[7] Stiles, V. H., James, I. T., Dixon, S. J., \& Guisaola, I. N. Natural turf surfaces: the case for continued research. Sports Medicine, 2009, 39, 65-84.

[8] Dumlop, J.Wear and care: assessing the benefits of synthetic surfaces. Stadia, 2001, 8, 74-76.

[9] Andersson, H., Ekblom, B., \& Krustrup, P. Elite football on artificial turf versus natural grass: movement patterns, technical standards, and player impressions. Journal of Sports Sciences, 2008, 26, 113-122.

[10] Hawkins, R. D., \& Fuller, C. W. A prospective epidemiological study of injuries in four English professional football clubs. British Journal of Sports Medicine, 33, 1999, 196-203.

[11] Fédération Internationale de Football Association. Grassroots manual. Zurich: FIFA Education and Technical Development, 2012.

[12] Aguiar, M., Gonçalves, B., Botelho, G., Lemmink, K., \&Sampaio, J. Footballers' movement behaviour during 2-, 3-, 4- and 5-a-side small-sided games.Journal of Sport Sciences, 2015, 33, 1259-1266.

[13] Little, T. Optimizing the use of soccer drills for physiological development. Strength and Conditioning Journal, 2009, 31, 67-74.

[14] Kawulich, B. Participant observation as a data collection method.Qualitative Social Research, 2005, 6, 43.

[15] Stein, H. \& Galili, I. The impact of an operational definition on the weight concept on student's understanding. International Journal of Science and Mathematics Education, 2015, 13, 1487-1515.

[16] Fleiss, J.L. (1981).Statistical methods for rates and proportions. ( $2^{\text {nd }}$ Edition). New York: John Wiley.

[17] OPTA (2012). Definitions OPTA and Pack Training 2012/13. OPTA Sportsdata.

[18] Alcántara, E., Gámez, J., Rosa, D., \& Sanchís, M. Analysis of the influence of rubber infill morphology on the mechanical performance of turf surfaces for soccer. Proceedings of the Institution of Mechanical Engineers, Part P: Journal of Sports Engineering and Technology, 2009, 223, 1-9.

[19] Impellizzeri, F. M., Rampinini, E., Castagna, C., Martino, F., Fiorini, S., \& Wisloff, U. Effects of plyometric training on sand versus grass on muscle soreness and jumping and sprinting ability in soccer players. British Journal of Sports Medicine, 2008, 42, 42-46.

[20] Fradua, L., Zubillaga, A., Caro, O., Fernandez-García, A. I., Ruiz-Ruiz, C., \& Tenga, A. Designing small sided games for training tactical aspects in soccer: extrapolating pitch sizes from full-size professional matches. Journal of Sports Sciences, 2013, 31, 573-581.

[21] Jones, S, \& Drust, B. Physiological and technical demands of 4 vs. 4 and 8 vs. 8 in elite youth soccer players. Kinesiology, 2007, 39, 150-156.

[22] De Baranda, P. S., Ortega, E., \& Palao, J. M. Analysis of goalkeepers defense in the World Cup in Korea and Japan in 2002. European Journal of Sport Science, 2008, 8, 127-134.

[23] Hughes, M., \& Franks, I. Analysis of passing sequences, shots and goals in soccer. Journal of Sports Sciences, 2005, 23, 509-514. 
[24] Zanetti, E. M. Amateur football game on artificial turf: Players' perceptions. Applied Ergonomics, 2009, 40, 485490.

[25] Casamichana, D., Castellano, J., \& Castagna, C. Comparing the physical demands of friendly matches and small-sided games in semi-professional soccer players. Journal of Strength and Conditioning Research, 2012, 26, 837-843.

[26] Binnie, M. J., Peeling, P., Pinnington, H., Landers, G., \& Dawson, B. Part 2: Effect of training surface on acute physiological responses following sport-specific training. Journal of Strength and Conditioning Research, 2012, 27, 1057-1066.

[27] Foster, J. B. Newer artificial turf appears safer for soccer players. Biomechanics, 2007, 14, 9-10.

[28] Orchard, J. Is there a relationship between ground and climatic conditions and injuries in football? Sports Medicine, 2002, 32, 419-432.

[29] DiMichele, R., DiRenzo, A. M., Ammazzalorso, S., \& Merni, F. Comparison of the physiological responses to an incremental running test on treadmill, natural grass, and synthetic turf in young soccer players. Journal of Strength and Conditioning Research, 2009, 23, 939-945.

[30] Aughey, R. Australian football player work rate: evidence of fatigue and pacing? International Journal of Sports Physiology and Performance, 2010, 5, 394-405.

[31] Ford, K., Manson, N., Evans, B., Myer, G., Gwin, R., \& HeiSD, R. Comparison of in-shoe foot loading patterns on natural grass and synthetic turf. Journal of Science and Medicine in Sport, 2006, 9, 433-440.

[32] Meyers, M., \& Barnhill, B. Incidence, causes, and severity of high school football injuries on FielSDurf vs. natural grass: A 5-year prospective study. American Journal of Sports Medicine, 2004, 32, 1626-1638.

[33] McNitt, A. S., Petrunka, D., \& Hardness, S. Evaluation of playing surface characteristics of various in filled systems. Evaluation, 2007, 9, 20-32.

[34] Villwock, M. R., Meyer, E. G., Powell, J. W., Fouty, A. J. \& Haut, R. C. Football playing surface and shoe design affect rotational traction. American Journal of Sports Medicine, 2009, 37, 518-525.

[35] Jastrzebski, Z., Bichowska, M., Rompa, P., Radziminski, L., \& Dargiewicz, R. Influence of different type of surfaces on the results of running speed tests in young soccer players. Central European Journal of Sport Sciences and Medicine, 2014, 5, 5-14.

[36] Lawton, G. Pitch battle over artificial grass. New Scientist, 2005, 2502, 35-37.

[37] Ekstrand, J., Timpka, T., \& Hagglund, M. Risk of injury in elite football played on artificial turf versus natural grass: a prospective two-cohort study. British Journal of Sports Medicine, 2006, 40, 975-980.

Citation: Jorge Diaz-Cidoncha Garcia. "Analysis of Offensive Technical Actions on Different Playing Surfaces in 5v5, 7v7 and 9v9 Sided Games in Soccer." International Journal of Sports and Physical Education (IJSPE), vol 3, no. 4, 2017, pp. 47-57. doi:http://dx.doi.org/10.20431/2454-6380.0304008.

Copyright: (C) 2017 Authors. This is an open-access article distributed under the terms of the Creative Commons Attribution License, which permits unrestricted use, distribution, and reproduction in any medium, provided the original author and source are credited. 